\title{
Balkanologie
}

Balkanologie Revue d'études pluridisciplinaires

Vol. IX, n' 1-2 | 2005

Volume IX Numéro 1-2

\section{De Waele (Jean-Michel), Husting (Alexandre), éds., Sport, politiques et sociétés en Europe centrale et orientale}

Bruxelles : Éditions de l'Université de Bruxelles, 2005, 166 pages

Taline Ter Minassian

\section{CpenEdition}

Journals

Édition électronique

URL : http://journals.openedition.org/balkanologie/1995

DOI : 10.4000/balkanologie.1995

ISSN : 1965-0582

Éditeur

Association française d'études sur les Balkans (Afebalk)

Édition imprimée

Date de publication : 1 décembre 2005

ISSN : 1279-7952

\section{Référence électronique}

Taline Ter Minassian, « De Waele (Jean-Michel), Husting (Alexandre), éds., Sport, politiques et sociétés en Europe centrale et orientale », Balkanologie [En ligne], Vol. IX, n 1-2 | 2005, mis en ligne le 14 janvier 2010, consulté le 17 décembre 2020. URL : http://journals.openedition.org/balkanologie/1995 ; DOI : https://doi.org/10.4000/balkanologie.1995

Ce document a été généré automatiquement le 17 décembre 2020.

(c) Tous droits réservés 


\section{De Waele (Jean-Michel), Husting (Alexandre), éds., Sport, politiques et sociétés en Europe centrale et orientale}

Bruxelles : Éditions de l'Université de Bruxelles, 2005, 166 pages

Taline Ter Minassian

\section{RÉFÉRENCE}

De Waele (Jean-Michel), Husting (Alexandre), éds., Sport, politiques et sociétés en Europe centrale et orientale, Bruxelles : Éditions de l'Université de Bruxelles, 2005, 166 p.

1 Sous ce titre générique associant mystérieusement une réalité supposée singulière du sport à la pluralité indéfinie des politiques et des sociétés en Europe centrale et orientale, apparaît l'un de ces ouvrages collectifs ficelés hâtivement par des éditeurs visiblement plus soucieux d'ajouter un titre supplémentaire à la liste déjà impressionnante de leurs publications que d'apporter à leurs lecteurs une réflexion étayée sur la sociologie politique du sport. Tous deux sont issus de la prestigieuse Université Libre de Bruxelles (ULB) : Jean-Michel de Waele a dirigé un certain nombre d'ouvrages collectifs portant essentiellement sur les partis politiques en Europe centrale et orientale tandis qu'Alexandre Husting, spécialiste des politiques publiques du sport, s'est beaucoup intéressé à la contribution du sport dans l'élaboration d'une «citoyenneté européenne». Sans préciser l'origine de cet ouvrage - une journée d'études, un colloque? dans quel cadre? - les éditeurs déplorent, dans leur introduction, que le sport, "fait social total», soit demeuré largement ignoré de la science politique. Pour autant, aucune problématique n'est véritablement posée faute d'une définition préalable du sport et d'une justification pertinente de l'espace envisagé. Après une introduction comportant certaines lourdeurs, le lecteur devra surmonter son appréhension pour aborder le corps de l'ouvrage composé de sept contributions de qualité inégale. Michel Raspaud et Radu Ababei abordent le thème de 
l'organisation et de l'économie $d u$ football roumain depuis la fin du régime communiste. Mais comment comprendre les mutations du milieu du football intervenues dans les années 1990 sans une présentation détaillée de ce milieu à l'époque communiste? Certes, les auteurs indiquent (p.25) qu'à l'époque du communisme roumain, «le championnat de football fonctionnait suivant la logique d'un système informel tout à l'avantage des clubs appartenant aux grands corps de l'État que sont l'Armée et la Police, lesquels, par des réseaux d'influence et la corruption, tiraient avantage sportif de leur domination sur les sphères politiques et la société » mais ne livrent aucune précision supplémentaire. En abordant l'influence de la transformation du régime politique en Pologne sur le fonctionnement des clubs sportifs, Andrzej Smolen propose une étude qui comporte une foule d'informations notamment sur l'orientation sportive des organisations politiques de l'entre-deuxguerres comme l'Union des associations sportives des travailleurs (liée au Parti socialiste polonais, PPS), l'Association de gymnastique Sokol (liée à la démocratie nationale) ou encore l'Association de tir Strzelec. À l'époque communiste, les représentants du parti, et en particulier les directeurs d'usines, semblent exercer leur patronage sur les clubs sportifs. Ainsi, le sociologue peut-il envisager ces conseils patronaux exerçant leur tutelle sur les clubs sportifs comme d'authentiques "corps sociaux" "dont les membres, dans le cas des clubs interentreprises ou municipaux, étaient le plus souvent des directeurs des établissements industriels les plus importants dans les diverses régions, des gouverneurs de ville et des personnes qui remplissaient des fonctions importantes dans les organes municipaux et provinciaux du PZPR » (p. 45). Véritable micro-société, l'entreprise socialiste trouve un prolongement dans les clubs sportifs et l'économie informelle qu'ils ne manquent pas de susciter. Cette situation évolue naturellement durant les années 1980 mais l'article n'aborde pas précisément des thèmes que l'on aimerait voir développés comme le rôle de l'Église catholique, de Solidarnosc ou même l'évolution des clubs sportifs aux chantiers Lénine de Gdansk qui aurait probablement fourni une étude de cas intéressante. En abordant le rôle du sport dans la construction de l'identité nationale lituanienne, Ingvaras Butautas et Rasa Čepaitiene, font apparaître le sport parmi les composantes de l'identité nationale en Lituanie où le basket fut longtemps élevé au rang de " religion nationale ». Ici, les auteurs abordent davantage le rôle des victoires sportives dans le renforcement des identités nationales, le cas lituanien étant intéressant de ce point de vue car dans le contexte soviétique, l'identité nationale s'est maintenue. Par exemple, le recrutement de l'équipe de football s'est accompli en Lituanie sur une base nationale comme en Géorgie ou en Arménie, ces cas étant à l'opposé du Kazakhstan dont l'équipe ne comptait qu'à peine $30 \%$ de sportifs kazakhs. L'article mentionne ensuite la portée politique des manifestations à l'issue de matchs comme celle des supporters de l'équipe de football Žalgiris de Vilnius en 1977 qui s'inscrit dans un contexte de contestation sensible en Lituanie depuis 1972 lorsqu'un étudiant de Kaunas s'était volontairement immolé en signe de protestation contre l'occupation soviétique. Dans une contribution dont le titre rappelle les mots d'ordre des hajduks (haïdouks) bulgares, «fidèles jusqu'à la mort », Maria Iliycheva analyse le nationalisme bulgare à travers le discours des supporters. Dans le style du langage guerrier propre à la compétition sportive, elle met en évidence les stéréotypes attachés à la désignation des adversaires, notant ainsi d'intéressantes réminiscences historiques: les autorités grecques du sport sont qualifiées de «byzantines » tandis que les équipes de football et les supporters croates sont désignés comme des Oustashis... Abordant le «cas symptomatique » de l'ex- 
Yougoslavie, Srdjan Vrcan aborde la seconde partie des années 1980. Malheureusement, le style déplorable de cet article ne permet pas de comprendre dans son essence le "symptôme » du football yougoslave. À propos de la politisation du milieu du football, l'auteur écrit par exemple que (sic) «cette politisation représente l'évolution d'une politisation, plutôt douce, sporadique et partielle du football par le socialisme yougoslave en crise, dans des formes atténuées, vers une politisation radicale et totale du football dans une direction opposée et dans un mode politique et idéologique nationaliste pendant la transition postsocialiste» (p.102) et que (sic) «la politisation transitionnelle du football a mené à son instrumentalisation politique quasi totale, ainsi qu'à une instrumentalisation footballistique de la politique d'aujourd'hui »(p.103). Philippe Chassagne et Kolë Gjeloshaj présentent la seule contribution synthétique portant sur les liens entre le sport et les «milieux d'affaires» dans les Balkans. L'implication des hommes d'affaires et des réseaux mafieux, la corruption, la criminalité déterminent les liens entre groupes de sportifs et milieux criminels. On voit apparaître ici une esquisse de typologie faisant intervenir une criminalité «traditionnelle », puis la criminalité économique à partir des années 1960 auxquelles il convient d'ajouter les sportifs et «bandits » anciens de la guerre d'Afghanistan... sans compter les cas de reconversion d'anciens sportifs dans les milieux criminels. Ainsi, après la chute du régime communiste en Bulgarie, bon nombre de sportifs - lutteurs, haltérophiles, boxeurs, adeptes de l'aviron etc. - se sont lancés dans des activités illicites cherchant notamment le contrôle du marché des paris. Le cas de Željko Ražnatović dit Arkan, bras armé du régime de Slobodan Milosevic, est présenté comme un cas d'école: son intervention dans le milieu du football serbe correspondait à la volonté de canaliser les groupes de supporters violents afin de créer une milice paramilitaire, la Garde des volontaires serbes. Par la suite, les intrigues d'Arkan dans le milieu sportif, épaulé par son épouse, la célèbre chanteuse Ceca, mettent clairement en évidence l'investissement du secteur sportif par les différents réseaux de la criminalité dans les Balkans. Clôturant l'ouvrage, la contribution d'Alexandre Husting consacrée aux sportifs professionnels originaires des PECO dans le football européen, ne présente pas, en dépit de nombreux tableaux et graphiques, une vue synthétique de la question. On retiendra cependant, que c'est surtout en Allemagne et en Autriche qu'évolue le plus grand nombre de footballeurs ressortissants des PECO dans les clubs de première division. Un tel ouvrage rédigé dans un style constamment pesant mêlant maladroitement le jargon de la sociologie à la terminologie sportive produit un effet plutôt déconcertant. Outre le manque de rigueur méthodologique - on aurait attendu par exemple une distinction élémentaire entre la pratique sportive et la haute compétition - il manque à l'évidence dans cet ouvrage des chapitres essentiels de la relation historique entre sport et politique dans l'espace envisagé : il s'agit de l'URSS et de la RDA dont le rôle central a été visiblement oublié. 